\title{
Control over the processing of the opponent's gaze direction in basketball experts
}

\author{
Matthias Weigelt $^{1}$ - Iris Güldenpenning ${ }^{1}$ Yvonne Steggemann-Weinrich ${ }^{1}$. \\ Mustafa Alhaj Ahmad Alaboud ${ }^{1}$ - Wilfried Kunde ${ }^{2}$
}

Published online: 19 August 2016

(C) Psychonomic Society, Inc. 2016

\begin{abstract}
Basketball players' responses to an opposing players' pass direction are typically delayed when the opposing player gazes in another than the pass direction. Here, we studied the role of basketball expertise on this, the so-called head-fake effect, in three groups of participants (basketball experts, soccer players, and non-athletes). The specific focus was on the dependency of the head-fake effect on previous fake experience as an index of control over the processing of task-irrelevant gaze information. Whereas (overall) the head-fake effect was of similar size in all expertise groups, preceding fake experience removed the headfake effect in basketball players, but not in non-experts. Accordingly, basketball expertise allows for higher levels of control over the processing of task-irrelevant gaze information.
\end{abstract}

Keywords Gaze direction · Expertise in sports · Information processing $\cdot$ Head fake

\section{Introduction}

The interaction between two or more athletes in most competitive sports, such as soccer, basketball or team handball, requires the successful competitor to discriminate deceptive from non-deceptive actions of the opponent. Evidence for superior performance of expert athletes in deception tasks has been provided in a number of previous studies, using the

Matthias Weigelt

matthias.weigelt@uni-paderborn.de

1 Department of Sport and Health, University of Paderborn, Warburger Str. 100, 33098 Paderborn, Germany

2 Department of Psychology, Würzburg University, Röntgenring 11, 97070 Würzburg, Germany expert-novice approach. For example, domain-specific expertise enables athletes to better anticipate the direction of penalty kicks in soccer (Smeeton \& Williams, 2012) and the direction of ground strokes in tennis (Rowe, Horswill, Kronvall-Parkinson, Poulter, \& McKenna, 2009), to better predict a change of running direction of a rugby player with and without deceptive movement (Jackson, Warren, \& Abernethy, 2006), and to recognize whether a team handball player throws or fakes a throw at the handball penalty shot (Cañal-Bruland \& Schmidt, 2009; Cañal-Bruland, van der Kamp, \& van Kesteren, 2010) or whether a basketball player passes the ball or fakes a pass to the teammate (Sebanz \& Shiffrar, 2009).

Many studies revealed superior expert performance in experimental designs using non-speeded responses as the dependent measure (i.e., in discrimination, recognition, and prediction tasks). This is perfectly appropriate when it comes to study (non-)experts' perceptual processing of fake actions. However, many competitive sports require not only the perceptual processing of fake actions, but motor responses towards these actions, mostly on a time scale of a few hundred milliseconds. Consequently, experimental designs using speeded responses may provide further insight into the question whether, and under which conditions, fake actions impact experts and non-experts motor performance.

The impact of time pressure on expert performance in deception tasks has been considered in only a few studies (Dicks, Button, \& Davids, 2010; Mori \& Shimada, 2013; Ripoll, Kerlirzin, Stein, \& Reine, 1995). These show, for example, that soccer goalkeepers save fewer penalty kicks in deceptive trials than in non-deceptive trials (Mori \& Shimada, 2013) and that French boxers produce more false alarms to a fake attack (Ripoll et al. 1995). Thus, there is some evidence that the performance of expert athletes also suffers under time pressure when they are confronted with a 
deceptive action. While appreciating these previous findings on expert-novice differences in discriminating deceptive from non-deceptive actions coming from studies using either speeded or non-speed responses, still little is known about why these actions work in the first place. That is, the cognitive mechanisms underlying deception in sports have not been fully uncovered by more systematic investigations using the inferential tools of experimental psychology.

It seems plausible that the cognitive mechanisms underlying the processing of deceptive information depend of the kind of deceptive action. Jackson et al. (2006) differentiated between two different categories of deception: On the one hand, an athlete might attempt to disguise his true action intention. To disguise the opponent means that the actor tries to minimize any informative cues of the action that he/she intends to perform. An attacking volleyball player, for example, might for as long as possible try to hide whether he/she wants to smash or lob the ball (Güldenpenning, Steinke, Koester, \& Schack, 2013). The challenge for the opponent is to infer the true action intention based on the reduced information available. On the other hand, an athlete performing a deceptive action might provide misleading information, resulting in an incorrect judgment. A popular example is the head fake in basketball, when, for example, a basketball player looks to the left while passing the ball to the right. The challenge for the opponent here is to focus on the relevant information (i.e., pass direction) and to ignore the conflicting information (i.e., gaze direction). The present manuscript focuses on the latter category of deception and examines the role of domain-specific athletic expertise on the processing of head fakes in sports.

Head fakes in sports were examined in a paradigmatic way by Kunde, Weigelt and colleagues (Alhaj Ahmad Alaboud, Steggemann, Klein-Soetebier, Kunde, \& Weigelt, 2012; Kunde, Skirde, \& Weigelt, 2011). These authors used the head fake in basketball as their standard model of a deceptive action. In the procedure of their experiments, participants were presented with static pictures of a basketball player performing chest passes to the left or right, while looking into the same (no head fake = pass-head congruence) or different (head fake $=$ pass-head incongruence) direction. They were instructed to respond to the pass direction for as fast and as accurately as possible, while ignoring the player's gaze direction. Responses were generally slower and more error prone for incongruent pass-head orientations, signifying the hadefake effect in basketball. The basic head-fake effect was independent of the response speed, the presence of a head fake in the immediately preceding trial, and practice with the task (Kunde et al. 2011, Experiment 1). However, the size of the head-fake effect can be influenced by a number of factors: It has been shown that the head-fake effect is modulated by the frequency with which head fakes are presented across an experiment, with the effect being larger the less frequent a head fake is presented and (vice versa) smaller the more often it occurs (Alhaj Ahmad Alaboud et al. 2012). Also, the presentation of dynamic displays, as compared to static stimuli, results in larger head-fake effects (Alhaj Ahmad Alaboud, Güldenpenning, Steggemann-Weinrich, Kunde, \& Weigelt, in press, Experiment 1). Interestingly, the complexity of the response does not influence the head-fake effect. That is, the effect is of similar size, irrespective of whether participants simply pressed buttons with their index fingers or if they responded with a whole-body movement, mimicking complex defensive basketball moves (Alhaj Ahmad Alaboud et al. in press, Experiment 2).

Two aspects of the data suggest that the head-fake effect originates from the perceptual processing of conflicting information of the relevant (pass direction) and irrelevant (gaze direction) stimulus features, but not from response selection and motor-priming effects (Kunde et al. 2011, Experiments 2-6). First, manipulations of the perceptual quality of the stimuli did have an impact on the fake effect, whereas manipulations of the type and compatibility of motor responses (typically ascribed to response selection) did not. Second, high temporal overlap of the fake-response task with another capacitylimited task tended to reduce the effect as compared to low overlap, which according to the locus-of-slack logic also suggests a perceptual origin (for more details see Kunde et al. 2011). Therefore, Kunde et al. (2011) argued that head fakes are processed automatically during stimulus encoding and that the resulting S-S type interference cannot be actively suppressed by the observer, delaying all other processes following thereafter, such as response selection and response programming. Although the precise mechanisms of the head-fake effect have not yet been identified, two hypotheses can explain the perceptual processing locus: First, the effectiveness of head fakes in basketball may be based on some kind of an input selection problem, where it is harder to discriminate between the relevant stimulus feature (pass direction) and the irrelevant stimulus feature (gaze direction). Solving this S-S discrimination task takes time and thus delays the perceptual processing of the pass direction. Such an input selection problem does not arise under congruent conditions (i.e., no head fake) when pass and gaze direction coincide. Second, it may be based on attention capture by the irrelevant stimulus feature and thus, under incongruent conditions (i.e., head fake), on an erroneous shift of visual attention to the player's gaze. Accordingly, the perceptual processing of the relevant stimulus feature is delayed, because the re-orientation of visual attention from the player's gaze to the pass takes time.

Previous studies on head fakes relied on the performance of non-athletes (Kunde et al. 2011) or athletes without domainspecific expertise (Alhaj Ahmad Alaboud et al. 2012, in press). Therefore, it is still unknown whether and how athletic expertise shapes the impact of head fakes within the domain of expertise. The present study addresses this question for the 
processing of head fakes in basketball. Expert athletes may be as vulnerable to the fake gaze information as non-athletes are, if they attend to this information, or they may have developed strategies to reduce the effect of the head fake. In the latter case, we assume that there are two ways of how expertise may change the manner in which head fakes by the opponent are processed by the observer.

First, massive amounts of deliberate practice over many years might enable basketball players to discriminate relevant from irrelevant stimulus features better than athletes without basketball-specific expertise and non-athletes. As a result, basketball players may have developed cognitive mechanisms to generally suppress the conflicting information and are therefore less distracted by the head fake. This should show up as an overall reduced fake effect in basketball experts compared to non-experts.

Second, basketball experts might have developed higher degrees of control over the processing of irrelevant information (i.e., head orientation). Consequently, they might be able to give irrelevant information higher processing weights, where this information had turned out to be helpful before, and reduce processing weight, when the same information had turned out to be detrimental. Such flexibility has been assumed in many sorts of structurally equivalent interference paradigms, such as Stroop-, Simon-, or Eriksen-Tasks. A key indication for such flexibility is the conflict sequence effect (CSE), that is, larger congruency effects after a previous congruent event (typically in trial n-1) rather than an incongruent event (Gratton, Coles, \& Donchin, 1992). The idea is that irrelevant information facilitates processing of relevant information in congruent cases so that irrelevant information is given a higher processing weight shortly after congruent events, whereby the congruency effect increases in the next trial. Interestingly, the studies so far suggest that such flexibility is severely limited in basketball novices, because they did not consistently show the typical CSE pattern; that is, the fake effects are of equal size, independent of whether a fake or nonfake action had been processed before (Alhaj Ahmad Alaboud et al. 2012; Kunde et al. 2011). It will therefore be of particular interest to see whether the typical CSE pattern, which signifies processing flexibility, is re-established by basketball (and thus head-fake) experts compared to both athletes without domainspecific expertise and non-athletes.

\section{Methods}

\section{Participants}

Sixteen experienced basketball players (four females, mean age 24.4 years) took part in the experiment. The inclusion criteria into the group of basketball players were that these athletes were currently practicing their sport and that they have had regular training over the last 5 years. The mean training (and playing) experience was 14.1 years. They were players from the first (six players) and second division (one player) of the German Basketball League, as well as from higher regional teams (seven players).

Two groups (with 24 participants in each group) were tested as control groups: A group of soccer players (five females, mean age 22.5 years) served as an athletic control group without specific basketball expertise. Their mean training (and playing) experience was 16.6 years. They played in different leagues in Germany, ranging from regional leagues (Bezirksliga) up to the second division of the Bundesliga. In addition, a group of non-athletes (six females, mean age 23.5 years) without any specific athletic training and expertise was tested.

All participants reported normal or corrected-to-normal vision and had no knowledge of the expected outcome of this experiment. Each participant gave informed consent to participate. They were not paid for participation. The single experimental session lasted about $30 \mathrm{~min}$. All rights of the participants were protected, and all experiments were carried out according to the 1964 Declaration of Helsinki.

\section{Apparatus and stimuli}

Stimuli were presented and responses and response times (RTs) were recorded by an IBM-compatible personal computer with a 17-inch. VGA display. All stimuli were presented in color on a white background and were composed of a male basketball player with a ball in his hands, looking to the right or left, while passing the ball in the same or the opposite direction (see Fig. 1). Each response was a single key press on a standard computer keyboard. These were carried out with the index fingers of each hand, which rested on the "." key (right hand, for detection of movements to the right) and on the "X" key (left hand, for detection of movements to the left) on the outside of the bottom row of the keyboard.

\section{Procedure and design}

Participants were given written instructions to respond to the pass direction (body movement) as quickly and accurately as possible. The first block of 50 trials was considered as practice to familiarize participants with the experiment. During this time, the investigator stayed in the room to answer any questions. Data from this block were not evaluated. The practice block was followed by four test blocks of 100 trials each, which were separated by short breaks if participants wanted to rest. During each test block, the four possible stimulus combinations occurred equally often and in random order, resulting in a total of 200 congruent and 200 incongruent test trials for each participant across the whole experiment. Each trial began with the presentation of a central fixation cross. 

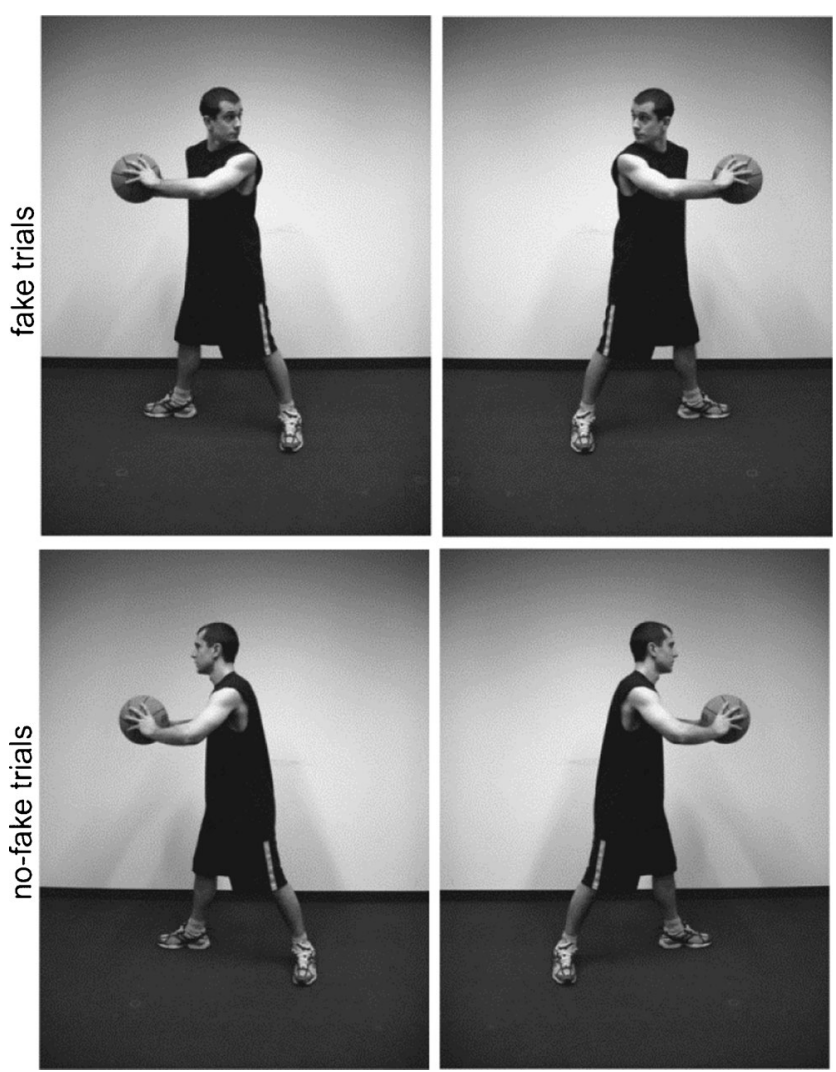

Fig. 1 Stimulus material: Participants were asked to respond to the pass direction of the depicted basketball player and to ignore gaze direction

After a fixed time interval of $250 \mathrm{~ms}$, the stimulus picture was presented and remained on the screen until a response was given. After the trial ended, participants received feedback about their answer. If there was an error, the word "Fehler" (German for "error") appeared on the screen for $500 \mathrm{~ms}$. If the answer was correct, the next trial began immediately with the appearance of the fixation cross.

\section{Results}

Response times were analyzed for correct responses and error rates for incorrect responses $(1.1 \%)$. Also, $0.1 \%$ of the data were excluded from further analyses, because RTs were either below $100 \mathrm{~ms}$ (considered as anticipations) or higher than $1,000 \mathrm{~ms}$ (considered as outliers).

\section{Sequential analysis}

Reaction times To find out if there are any differences between the groups in their ability to control over the processing of irrelevant information (i.e., head orientation), as would be signified by a CSE, the mean RTs were submitted to a mixed ANOVA with the factors fake (fake vs. non-fake action), fake in trial $n-1$ (fake vs. non-fake action), as repeated measures, and athletic expertise (basketball players, soccer players, nonathletes), as between participants factor. The modulation of the fake effect as a function of previous fake experience and expertise is shown in Fig. 2. The analysis of RTs revealed a significant main effect for the factor fake $[F(1,61)=46.61, p$ $\left.<.001 ; \mathrm{p}^{2}=.43\right]$. Moreover, the two-way interaction between fake and fake in trial $n-1$ reached significance $[F(1,61)=$ $\left.16.52, p<.001,{ }_{\mathrm{p}}^{2}=.21\right]$, as well as the three-way interaction between fake, fake in trial $n-1$, and athletic expertise $[F(2,61)=$ $\left.7.97, p<.001 ; \mathrm{p}^{2}=.21\right]$. No other main effect and none of the remaining two-way interactions were significant (all $p s>$ .10).

To examine differences in the modulation of the head-fake effect as a function of fakes in trial $n$ - 1 between the different groups of athletic expertise, a number of paired $t$-tests were conducted. For basketball players, there was a significant head-fake effect $(17 \mathrm{~ms})$ in trial $n$ when the previous trial was no head fake $[t(15)=5.00 ; p<.001]$, but the head-fake effect was absent $(-0.6 \mathrm{~ms})$ when a head fake was shown in the previous trial $(p>.70)$. For soccer players, the head-fake effect was significant irrespective of whether the immediately preceding trial $n-1$ was a head fake (4 ms) $[t(23)=2.3 ; p<$ $.05)]$ or was not a head fake $(7 \mathrm{~ms})[t(23)=3.6 ; p<.01]$. Similarly, for non-athletes, there was a significant head-fake effect $(7 \mathrm{~ms})$ in trial $n$, if the previous trial was not a head fake $[t(23)=2.5 ; p<.05]$, and there was a significant head-fake effect $(6 \mathrm{~ms})$, if the previous trial was a head fake $[t(23)=4.0$; $p<.001]$. The fake effect after regular passes in trial $n-1$ was larger in basketball experts compared to soccer players [ $(t(38)$ $=2.79 ; p<.01)]$, as well as non-athletes $[(t(38)=2.22 ; p<$ $.05)]$.

Error rates The modulation of the head-fake effect was also analyzed for error rates in a similar way (see Fig. 2). The ANOVA did not reveal a significant main effect and none of the two-way interactions were significant. However, the threeway interaction of the factors fake, fake in trial $n-1$, and athletic expertise was significant $\left[F(2,61)=6.72, p<.01 ; \mathrm{p}^{2}=\right.$ .18]. The head-fake effect in trial $n$ as a function of trial $n-1$ in dependence of athletic expertise was (again) examined by calculating a number of paired $t$-tests within each expertise group. For basketball players, there was a significant headfake effect $(1.2 \%)$ in trial $n$ if the previous trial was no head fake $[t(15)=3.10 ; p<.01]$, but there was no head-fake effect $(-0.7 \%)$ if the previous trial was a head fake $(p>.10)$. No such modulation of the head-fake effect was found for the other two groups (all $p \mathrm{~s}>.10$ ).

\section{Effects of practice}

Reaction times To examine practice-related changes in RTs across the experiment, data were separated into mini-blocks of 100 trials (see Fig. 3). An ANOVA with the within-subject 


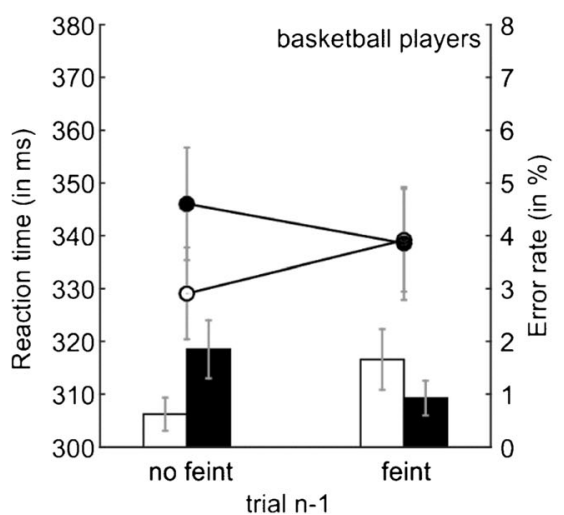

Fig. 2 Sequential modulation of the head-fake effect, separately for basketball players (left column), soccer players (middle column), and non-athletes (right column). Lines with unfilled circles represent response times to stimuli with congruent pass-head orientations, whereas lines with filled circles represent responses to stimuli with

factor of pass-head congruency (congruent vs. incongruent) and block (1-4) and the between-subjects factor athletic expertise (basketball players vs. soccer players vs. non-athletes) was calculated. There was a main effect of pass-head congruency $\left[F(1,61)=43.910, p<.001 ; \eta_{\mathrm{p}}{ }^{2}=.42\right]$ and a two-way interaction of block and athletic expertise $[F(6,183)=2.831$, $\left.p<.05 ; \mathrm{n}_{\mathrm{p}}{ }^{2}=.09\right]$. The main effect of pass-head congruency signifies the basic head-fake effect. The two-way interaction yields different RT patterns as a function of practice with the task and athletic expertise: RTs became faster for basketball players across the experiment, they became slower for soccer players, and they remained stable for non-athletes. Importantly, pass-head congruency did not interact with any other factor, indicating that the head-fake effect was not reduced through practice across the experiment. None of the other interactions was significant.

Error rates Effects of practice were also analyzed for error rates (see Fig. 3). The ANOVA did not reveal any significant main effects for the within-subjects factors and for the interactions (all $p s>.10$ ). Also the between subjects factor athletic expertise did not reach significance $(p>.08)$.

\section{Discussion}

The more general aim of the present study was to shed light on the role of domain-specific athletic expertise on the processing of deceptive actions in sports. Previously, Kunde et al. (2011) suggested that head fakes in basketball are processed automatically and cannot be suppressed by the observer. The data of their experiments and of other studies (Alhaj Ahmad Alaboud et al. 2012, in press), however, were based upon the performance of either non-athletes or of athletes without domainspecific expertise. Therefore, we investigated the effectiveness of the head fake in basketball and asked the question if domain-specific athletic expertise reduces the head-fake effect in basketball players, as compared to soccer players without

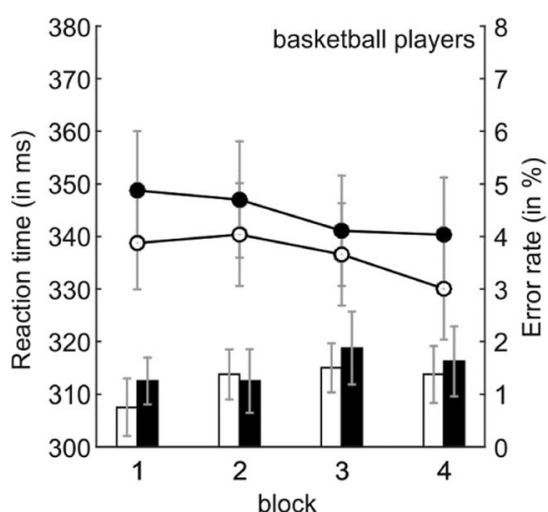

Fig. 3 Effects of practice: Reaction times as a function of practice block (100 trials each) and pass-head congruency, separately for basketball players (left column), soccer players (middle column), and non-athletes (right column). Lines with unfilled circles represent response times to stimuli with congruent pass-head orientations, whereas lines with filled
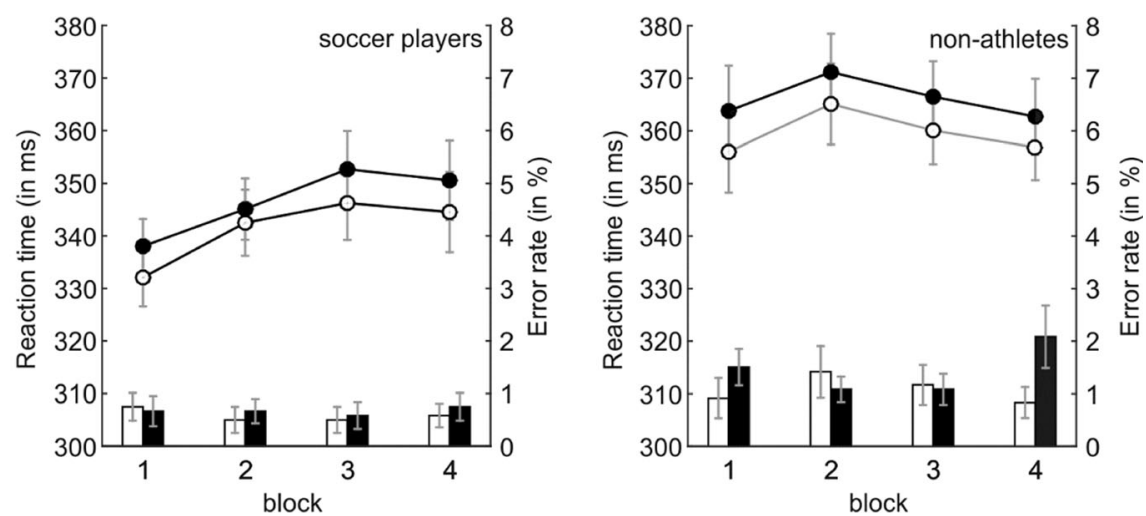

circles represent responses to stimuli with incongruent pass-head orientations. White bars illustrate response errors to stimuli with congruent pass-head orientation and black bars illustrate response errors to stimuli with incongruent pass-head orientation. Error bars represent standard errors 
domain-specific expertise (but with athletic training) and nonathletes (without athletic training and without expertise).

On first sight, the results are straightforward: Participants' responses were generally slower for incongruent pass-head orientations, signifying the head-fake effect. This finding is in line with previous studies demonstrating the delayed processing of head fakes in basketball (Alhaj Ahmad Alaboud et al. 2012; Kunde et al. 2011). The head-fake effect was found to be overall independent of practice with the task and, most importantly, independent of athletic expertise. That is, the head fake had a similar impact (i.e., the size of the head-fake effect was not reduced) on the performance of basketball players, as compared to soccer players and nonathletes. This pattern of results supports previous studies showing that performance of expert athletes suffers under time pressure, when they are confronted with a deceptive action (Dicks et al. 2010; Mori \& Shimada, 2013; Ripoll et al. 1995).

When taking a closer look at the data, however, the sequential analysis revealed that the effect disappeared in trial $n$ in basketball experts, but not in soccer players and non-athletes, when a head fake was presented in the immediately preceding trial $n-1$. Conversely the fake effect increased relative to nonexperts, when the gaze direction was congruent with the required response in the preceding trial. This finding is most interesting as it points to the (exceptional) ability of basketball players to circumvent interference from irrelevant gaze direction, when this had been proven to be detrimental in the previous trial, and conversely to process irrelevant information, when it had turned out to be helpful. This sequential modulation of the head-fake effect in basketball players is similar to what has been reported for structurally similar effects, such as the Eriksen effect (Gratton et al. 1992), the Simon effect (Stürmer, Leuthold, Soetens, Schröter, \& Sommer, 2002), or the Stroop effect (Kerns et al. 2004). The cognitive mechanisms for such a conflict adaptation are under debate (e.g. Hommel, Proctor, \& Vu, 2004). Two not mutually exclusive hypotheses have been put forward and supported to some extent respectively: Task-irrelevant information (i.e., misleading gaze direction) is suppressed or the focus of attention to the task-relevant information (i.e., informative/useful pass direction) is amplified in the trial following an information conflict (Kunde \& Wühr, 2006).

Thus, massive amounts of deliberate practice seem to provide basketball players either with the exceptional skill to suppress the gaze direction of the opponent or to focus attention to those body parts executing the intended passing action, after they had just experienced a head fake. Most interestingly, conflict adaptation to head fake repetition appears to be a domain-specific skill of basketball players, which is most likely based on deliberate practice in their sport, because soccer players with similar amounts of athletic training (even more years of practice in the present sample), but different athletic expertise, and non-athletes did not show such sequential modulations in their response behavior in the present study. From a practical point of view, the effectiveness of head fakes is reduced, when a basketball player has just encountered a head fake a moment before. Thus, the head fake loses its impression on the opponent when it is repeated. Basketball experts should therefore not repeat the same head fake immediately under competitive play. It also seems to lose its impact when it is used too often over the course of the game, which can be inferred from the results of Alhaj Ahmad Alaboud et al. (2012), who manipulated the overall frequency of head fake presentations. The head-fake effect was weaker the more head fakes were shown. Therefore, basketball players should acquire a greater repertoire of deceptive actions to be successful under competitive play.

The present study provides further evidence for the effectiveness of head fakes in basketball. Domain-specific athletic expertise based on deliberate practice, however, can lead to strategic conflict adaptation (as signified by the CSE) in basketball players, when head fakes are repeated. The cognitive mechanisms underlying this strategic conflict adaptation and, thus, the question of whether basketball players are able to suppress the misleading gaze direction or to focus their attention to the relevant body part to successfully respond to the passing action should be investigated in future studies.

Acknowledgments The authors thank Sarah Julia Büker and Lukas Jakobsmeyer for their help with data acquisition.

\section{References}

Alhaj Ahmad Alaboud, M., Güldenpenning, I., Steggemann-Weinrich, Y., Kunde, W. \& Weigelt, M. (in press). Eine experimentelle Untersuchung des Blicktäuschungseffektes im Basketball unter quasi-realistischen Bedingungen [Deception in sports: An experimental study on the head fake effect in basketball under quasirealistic conditions.]. Sportwissenschaft.

Alhaj Ahmad Alaboud, M., Steggemann-Weinrich, Y., Klein-Soetebier, T., Klein-Soetebier, T., Kunde, W., \& Weigelt, M. (2012). Täuschungshandlungen im Sport. Eine experimentelle Untersuchung zur Wirkung der Häufigkeitsverteilung auf die Blicktäuschung im Basketball. [Deception in sports: An experimental study on the impact of different frequency distributions of head fakes in basketball]. Zeitschrift für Sportpsychologie, 19(3), 110-121.

Cañal-Bruland, R., \& Schmidt, M. (2009). Response bias in judging deceptive movements. Acta Psychologica, 130(3), 235-240.

Cañal-Bruland, R., van der Kamp, J., \& van Kesteren, J. (2010). An examination of motor and perceptual contributions to the recognition of deception from others' actions. Human Movement Science, 29(1), 94-102.

Dicks, M., Button, C., \& Davids, K. W. (2010). Availability of advance visual information constrains association-football goalkeeping performance during penalty kicks. Perception, 39(8), 1111-1124.

Gratton, G., Coles, M. G. H., \& Donchin, E. (1992). Optimizing the use of information: Strategic control of activation of responses. Journal of Experimental Psychology: General, 121(4), 480-506.

Güldenpenning, I., Steinke, A., Koester, D., \& Schack, T. (2013). Athletes and novices are differently capable to recognize feint and 
non-feint actions. Experimental Brain Research, 230(3), 333-343. doi:10.1007/s00221-013-3658-2

Hommel, B., Proctor, R. W., \& Vu, K.-P. L. (2004). A feature-integration account of sequential effects in the Simon task. Psychological Research, 68(1), 1-17.

Jackson, R. C., Warren, S., \& Abernethy, B. (2006). Anticipation skill and susceptibility to deceptive movements. Acta Psychologica, 123(3), 355371.

Kerns, J. G., Cohen, J. D., MacDonald, A. W., Cho, R. Y., Stenger, V. A., \& Carter, C. S. (2004). Anterior cingulate conflict monitoring and adjustments in control. Science, 303(5660), 1023-1026.

Kunde, W., Skirde, S., \& Weigelt, M. (2011). Trust my face: Cognitive factors of head fakes in sports. Journal of Experimental Psychology: Applied, 17(2), 110-127. doi:10.1037/A0023756

Kunde, W., \& Wühr, P. (2006). Sequential modulations of correspondence effects across spatial dimensions and tasks. Memory \& Cognition, 34(2), 356-367. doi:10.3758/BF03193413

Mori, S., \& Shimada, T. (2013). Expert anticipation from deceptive action. Attention, Perception, \& Psychophysics, 75(4), 751-770.
Ripoll, H., Kerlirzin, Y., Stein, J.-F., \& Reine, B. (1995). Analysis of information processing, decision making, and visual strategies in complex problem solving sport situations. Human Movement Science, 14(3), 325-349.

Rowe, R., Horswill, M. S., Kronvall-Parkinson, M., Poulter, D. R., \& McKenna, F. P. (2009). The effect of disguise on novice and expert tennis players' anticipation ability. Journal of Applied Sport Psychology, 21(2), 178-185.

Sebanz, N., \& Shiffrar, M. (2009). Detecting deception in a bluffing body: The role of expertise. Psychonomic Bulletin \& Review, 16(1), 170-175.

Smeeton, N. J., \& Williams, A. M. (2012). The role of movement exaggeration in the anticipation of deceptive soccer penalty kicks. British Journal of Psychology, 103(4), 539-555.

Stürmer, B., Leuthold, H., Soetens, E., Schröter, H., \& Sommer, W. (2002). Control over location-based response activation in the Simon task: Behavioral and electrophysiological evidence. Journal of Experimental Psychology: Human Perception and Performance, 28(6), 1345-1363. 